\title{
Breve noticia sobre a hematita de Antonio Pereira
}

\author{
(2 figuras no texto e 1 quadro)
}

\author{
R. R. Franco
}

A hematita de Antonio Pereira (Minas Geraes, Brasil) apresenta-se com diversos aspectos sendo frequentes os aggregados granulares e microgranulares que se desaggregam com relativa facilidade; tambem frequentes os agrupamentos em roseta em que os cristaes são tabulares e acrescidos quasi parallelamente, (fig. I). Os cristaes isolados são muito raros.

A hematita desta procedencia é de coloração negra intensa, apresentando algumas vezes forte iridescencia. Devido ao seu brilho é geralmente conhecida pelo nome de "especularita".

Já foi estudada sob o ponto de vista morphologico e cristallographico por diversos autores, mas julgamos interessante apresentar, com este trabalho, o resultado de observações sobre alguns novos cristaes.

Os habitus por nós encontrados são: o tabular e o romboedrico, sendo que o primeiro é muito mais frequente. Nestes, os cristaes attingem algumas vezes $\mathrm{di}$ versos centimetros de diametros e apresentam-se com uma simetria pseudo-hexagonal (Quadro, figs. 1 e 2) bem caracteristica.

Entre os cristaes romboedricos é interessante notar que alguns mostram um

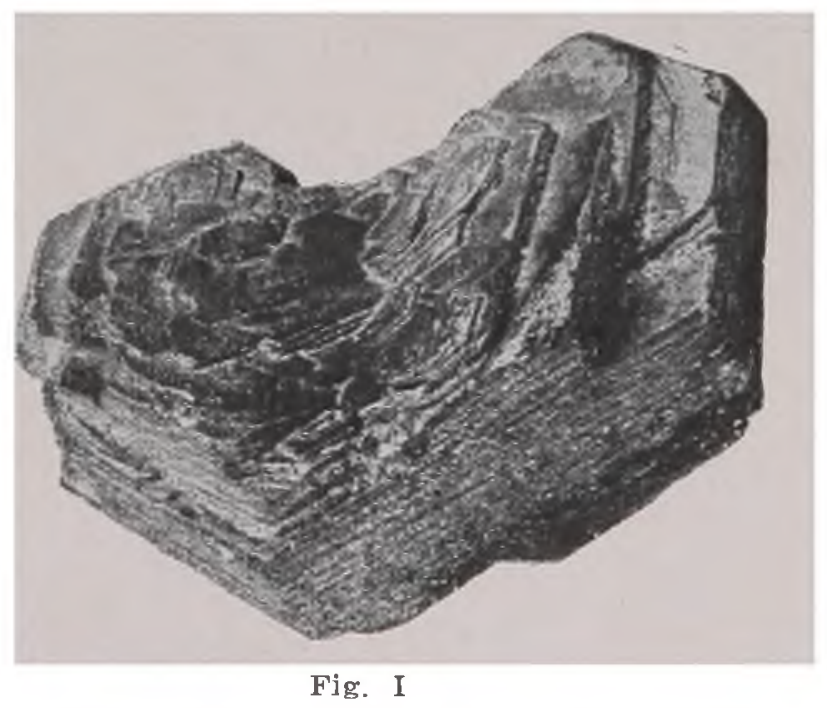
aspecto pseudo-cubico, porquanto as faces de romboedro funda- 
mental se apresentam muito e igualmente desenvolvidas (Quadro, fig. 3).

Damos a seguir as formas encontradas:

$$
\begin{array}{ll}
\mathrm{c}\{111\}=\{0001\} & \mathrm{g}\{411\}=\{3254\} \\
\mathrm{r}\{100\}=\{10 \overline{1} 1\} & \mathrm{i}\{51 \overline{1}\}=\{4265\} \\
\mathrm{r}^{\prime}\{221\}=\{01 \overline{1}\} & 0 \quad\{61 \overline{1}\}=\{52 \overline{7} 6\} \\
\mathrm{n}\{31 \overline{1}\}=\{22 \overline{4}\} & \mathrm{e}\{71 \overline{1}\}=\{62 \overline{8} 7\} \\
\mathrm{m}\{10 \overline{1}\}=\{11 \overline{2} 0\} & \mathrm{x}\{32 \overline{1}\}=\{13 \overline{4} 4\} \\
& \mathrm{k}\{20 \overline{1}\}=\{21 \overline{3} 1\} \\
\mathrm{s}\{32 \overline{2}\}=\{14 \overline{5} 3\}
\end{array}
$$

conforme a projecção annexa (Fig. II).

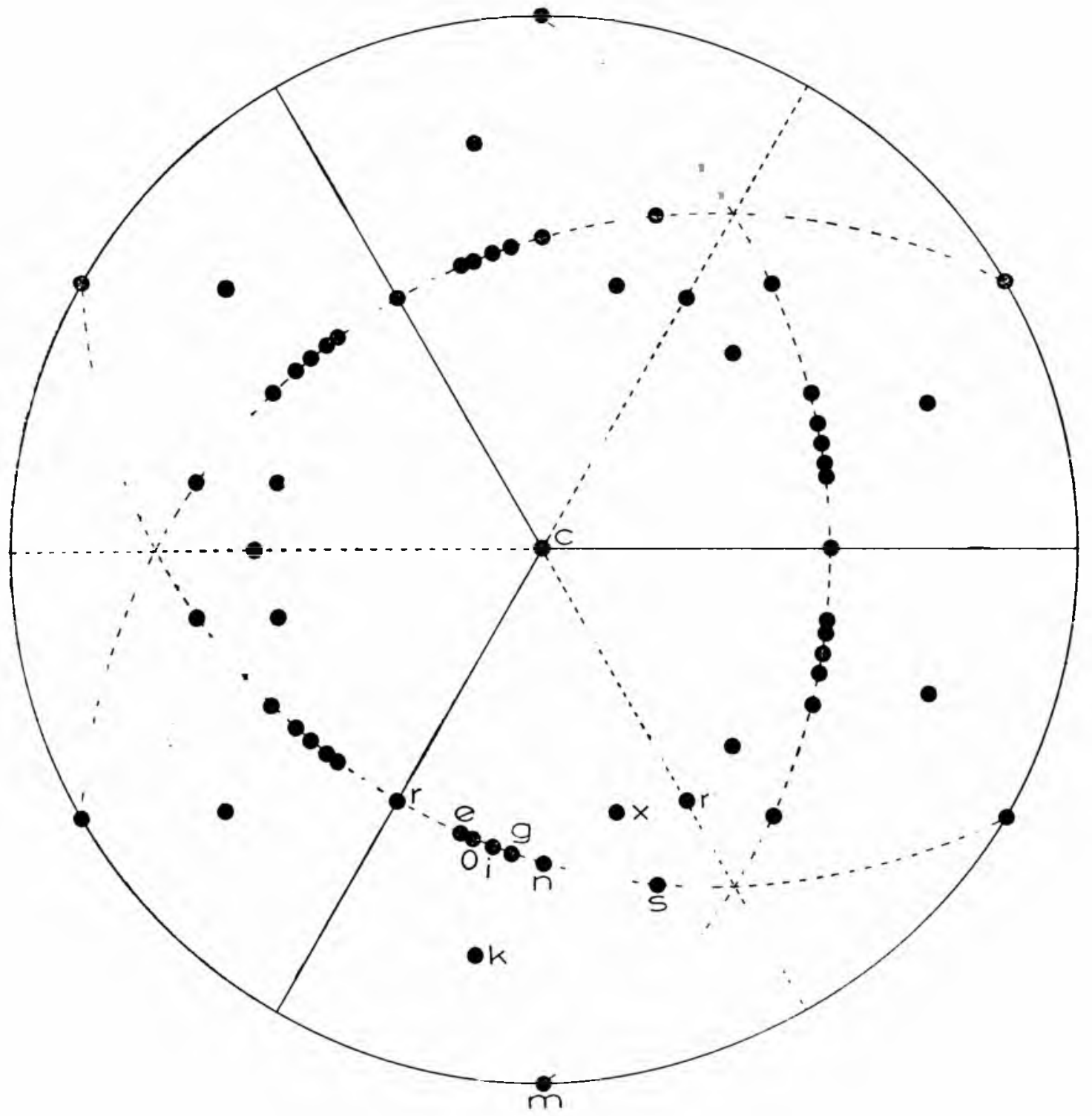

Fig. II 
As formas mais frequentes são $\{111\},\{100\},\{311\},\{511\}$ e $\{71 \overline{1}\}$ sendo que a $2 .^{\mathrm{a}}, 3 .^{\mathrm{a}}$ e a $5 .^{\mathrm{a}}$ foram encontradas sem excepção em todos os cristaes estudados. A face (100) do romboedro fundamental se apresenta commumente bem estriada dando reflexos multiplos.

O pinacoide (111) é sempre limpido e muito brilhante, sendo a face que mais se desenvolve.

As faces $(51 \overline{1})$ e $(71 \overline{1})$ nos cristaes estudados são relativamente bem desenvolvidas.

As outras faces são em geral pequenas e estreitas, não deixando entretanto de offerecer bellos reflexos.

Confirmamos aqui a existencia do escalenoedro inverso $\{32 \overline{1}\}$ descripto pela primeira vez por Dufet (1). Além da zona [111: $1 \overline{1} \overline{7}=3 \overline{4} 1]$, citada por este autor, conseguimos optimas medidas na zona $[02 \overline{1}: 100=012]$ a que tambem pertence a referida fórma.

Combinações encontradas.

Fig. $1-\{111\},\{311\},\{100\},\{51 \overline{1}\}$

Fig. $2-\{111\},\{31 \overline{1}\},\{100\},\{51 \overline{1}\},\{41 \overline{1}\},\{71 \overline{1}\}$ e $\{20 \overline{1}\}$

Fig. $3-\{20 \overline{1}\} \quad\{31 \overline{1}\},\{100\},\{71 \overline{1}\},\{10 \overline{1}\},\{32 \overline{1}\},\{22 \overline{1}\}$

Fig. $4-\{111\},\{31 \overline{1}\},\{100\},\{71 \overline{1}\},\left\{22^{\bar{z}}\right\}$

Utilisando o angulo entre as duas faces do romboedro fundamental, (100) e (010) de cujas medidas resultou para média $94^{\circ}$, vem como valor para a constante $\alpha=85^{\circ} 42^{\prime}$, correspondente, se quizermos fazer referencia á cruz bravaisiana do hexagonal, ao valor encontrado por Kokscharoff:

$$
\mathrm{a}: \mathrm{c}=1: 1,36557
$$

o qual tambem calculamos directamente partindo do angulo de (111) com (100) que é de $57^{\circ} 37^{\prime}$

Segue-se abaixo a tabella dos valores angulares medidos e calculados.

(1) M. H. DufeT - Description d'un cristal d'oligiste, Bulletin de la Societé Française de Minéralogie - 1.903, pag. 61. 


\section{ANGULOS}

\begin{tabular}{|c|c|c|c|c|c|c|}
\hline \multirow[b]{2}{*}{$c / r$} & \multirow[b]{2}{*}{$=$} & \multirow[b]{2}{*}{ (111) } & \multicolumn{2}{|r|}{ Medidos } & \multicolumn{2}{|c|}{ Calculados } \\
\hline & & & $(100)$ & $57^{\circ} 37^{\prime}$ & $57^{\circ}$ & $37^{\prime}$ \\
\hline c $n$ & $=$ & (111) & $(31 \overline{1})$ & $61^{\circ} \quad 12^{\prime}$ & $61^{\circ}$ & $13^{\prime}, 5$ \\
\hline $\mathrm{c} / \mathrm{i}$ & $=$ & (111) & $(51 \overline{1})$ & $58^{\circ} 23^{\prime}$ & $58^{\circ}$ & $24^{\prime}$ \\
\hline$r / r$ & $=$ & $(100)$ & $(010)$ & $94^{\circ}$ & $94^{\circ}$ & \\
\hline $\mathrm{n} n$ & $=$ & $(31 \overline{1})$ & $(13 \overline{1})$ & $51^{\circ} \quad 58^{\prime}, 5$ & $51^{\circ}$ & $59^{\prime}$ \\
\hline $\mathrm{n} r$ & $=$ & $(31 \overline{1})$ & $(100)$ & $26^{\circ}$ & $25^{\circ}$ & $59^{\prime}, 5$ \\
\hline $\mathrm{n}$ & $=$ & $(31 \overline{1})$ & $(711)$ & $14^{\circ} \quad 10^{\prime}, 5$ & $14^{\circ}$ & $11^{\prime}$ \\
\hline $\mathrm{x} / \mathrm{x}$ & $=$ & $(3 \overline{1} 2)$ & $(2 \overline{1} 3)$ & $22^{\circ} 37^{\prime}, 5$ & $22^{\circ}$ & $39^{\prime}$ \\
\hline n 0 & $=$ & $(31 \overline{1})$ & $(61 \overline{1})$ & $12^{\circ} 16^{\prime}, 5$ & $12^{\circ}$ & $18^{\prime}$ \\
\hline$n x$ & $=$ & $(31 \overline{1})$ & $(3 \overline{12})$ & $15^{\circ} \quad 3^{\prime}$ & $15^{\circ}$ & $3^{\prime}$ \\
\hline $\mathrm{n} / \mathrm{x}$ & $=$ & $(31 \overline{1})$ & $(2 \overline{1} 3)$ & $15^{\mathrm{o}} \quad 4^{\prime}$ & $15^{\circ}$ & $3^{\prime}$ \\
\hline $\mathrm{e}$ & $=$ & $(17 \overline{1})$ & $(02 \overline{1})$ & $18^{\circ} 39^{\prime}$ & $18^{\circ}$ & $45^{\prime}$ \\
\hline $\mathrm{m} / \mathrm{n}$ & $=$ & $(10 \overline{1})$ & (311) & $28^{\circ} 45^{\prime}$ & $28^{\circ}$ & $46^{\prime}, 5$ \\
\hline $\mathrm{c} / \mathrm{k}$ & $=$ & $(111)$ & $(20 \overline{1})$ & $76^{\circ} 29^{\prime}, 5$ & $76^{\circ}$ & $31^{\prime}$ \\
\hline$c / x$ & $=$ & (111) & $(3 \overline{12})$ & $54^{\circ} \quad 53^{\prime}$ & $54^{\circ}$ & $52^{\prime}$ \\
\hline $\mathrm{r} e$ & $=$ & $(100)$ & $(71 \overline{1})$ & $11^{\circ} 49^{\prime}$ & $11^{\circ}$ & $48^{\prime} 5$ \\
\hline
\end{tabular}




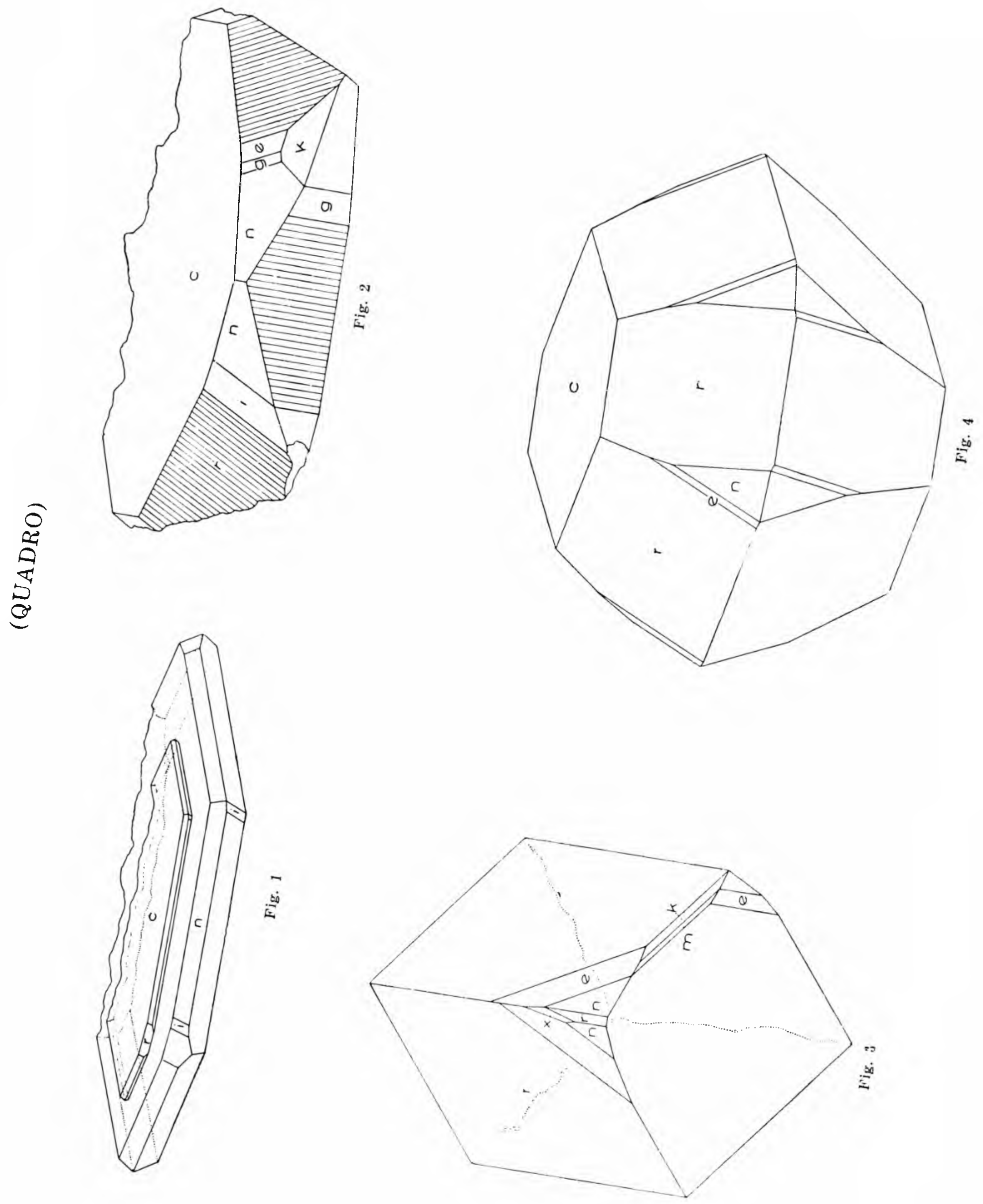





\section{SUMMARIUM}

\section{EX ANTONIO PEREIRA HAEMATITAE EXPEDITIO}

Ex Antonio Pereira haematita concretionibus apparet, infrequentibus crystallis singulis.

Magnitudo varia: nonnullis etiam octo centimetrorum amplitudo maxima est. Crystalla nigrantia, interdum iricoloria.

Habitus: rhomboëdricus ac tabularis secundum pinacoidium.

Quas reperimus formas:

$\mathrm{c}\{111\}, \mathrm{r}\{100\}, \mathrm{r}^{\prime}\{22 \overline{1}\} \mathrm{n}\{3 \overline{1}\}, \mathrm{m}\{10 \overline{1}\}, \mathrm{g}\{41 \overline{1}\}, \mathrm{i}\{51 \overline{1}\}$, $\mathrm{O}\{61 \overline{1}\}, \mathrm{e}\{71 \overline{1}\}, \mathrm{x}\{32 \overline{1}\}, \mathrm{k}\{20 \overline{1}\}, \mathrm{s}\{32 \overline{2}\}$

Constans : $\alpha=85^{\circ} 42^{\prime}$ 



\section{ERRATA DO "MINERALOGIA N. ${ }^{\circ} 2$ (1938)}

Pg. Linha Onde se lê:

30

30

3

$32 \quad 15 \quad\{713\}$

$32 \quad 16 \quad \mathrm{y}\{534\}$ ?

$32 \quad 20 \quad\{27 \overline{5}\}$

$32 \quad 21 \mathrm{H}\{14,4, \overline{3}\}: * \mathrm{H}_{1}\{14, \overline{3}, 4\} * \mathrm{~K}\{10, \overline{2}, 3\} *$

$32 \quad 22$

35

$35 \quad 2 \quad\{22,1, \overline{3}\}$

$35 \quad 13 \quad$ cito

$\begin{array}{lll}36 & 18 & \text { [132] }\end{array}$

$37 \quad 31 \quad$ outros identicos para media

$38 \quad 2 \quad\{201\}\{210\}$

$\begin{array}{lll}38 & 21 \quad \text { [21̄̄] }\end{array}$

$38 \quad 30 \quad$ Os valores

$39 \quad 4 \quad[100: 14 \overline{1}=01 \overline{4}]$

$39 \quad 14 \quad[\overrightarrow{12} 1]$

$3914 \quad[221: 010=102]$

$39 \quad 18 \quad[10 \overline{1}: 2 \overline{11}=153]$

$39 \quad 35 \quad\{4 \overline{1}\}$ e $\{4 \overline{11}\}$

$40 \quad 1 \quad[20 \overline{1}: 210=122]$

$40 \quad 7 \quad(10 \overline{10}):(32 \overline{54})-(211):(41 \overline{1})$

$40 \quad 23 \quad$ (1123)

$40 \quad 26 \quad\{524\}$

$40 \quad 27 \quad$ direitos

$41 \quad 12$ feitas as

$4119 \quad[210: 12 \overline{1}=1 \overline{2} 4]$

$41 \quad 25$

$41 \quad 28$

$41 \quad 29$

$42 \quad 5$

$42 \quad 6$

42 $[\overline{011}: 110=111]$ e $[112: 2 \overline{11}=\overline{1} 31]$

$$
(5,12, \overline{7}, 4)
$$

(17) $\overline{6} 2)$

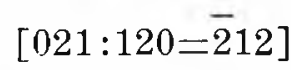

$(2,10,3)$

$[\overline{1} 43: 134=711]$
Leia-se:

$\{32 \overline{1}\}$

$\{1 \overline{12} 3\}$

$\{7 \overline{13}\}$

y $\{534\} *$ ?

$\{27 \overline{5}\} * ?$

$\mathrm{K} \nmid 10,3, \overline{2}\} *, \mathrm{~K}_{1}\{10, \overline{2}, 3\} * \mathrm{H}_{1}\{14 \overline{3}, 4\} *$ $\mathrm{L}\{16,5, \overline{3}\}$

$\{5 \overline{2} 2\}$

$\{22, \overline{1}, \overline{3}\}$

citamos

[1] 2 2]

cutros identicos obtendo para média $\{20 \overline{1}\} 2 \overline{10}\}$

[22-̄]

Alguns valores

$[100: 141=0 \overline{1} 4]$

[11르

[2]12:100 $=021]$

[11르: $2 \overline{1} 1=153]$

$\{4 \overline{1}\}$ e $\{4 \overline{1} 1\}$

[20̄1 :210 $=\overline{12} 2]$

$(10 \overline{10}):(32 \overline{5} 4)-(2 \overline{11}):(41 \overline{1})$

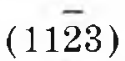

$\{52 \overline{4}\}$

directos

feitas sobre as

[210:021 $=1 \overline{24}$ ]

$[01 \overline{1}: 110=\overline{1} 11]$ e $[11 \overline{2}: 21 \overline{1}=\overline{1} 31]$

$(\overrightarrow{5}, 12, \overline{7}, 4)$

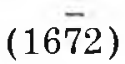

[021: $120=2 \overline{1} 2]$

$(\overline{2}, 10,3)$

[ $\overline{1} 43: \overline{1} 34=711]$ 


$\begin{array}{rrll}42 & 7 & {[221: 12 \overline{1}=4 \overline{1} 6]} & {[22 \overline{1}: \overline{1} 21=4 \overline{1} 6]} \\ 42 & 17 & (3,10,2) & (3,10, \overline{2}) \\ 45 & 2 & \{201\} & \{20 \overline{1}\} \\ 52 & 24 & 1 \mathrm{a} . & 2 \mathrm{a} . \\ 54 & 7 & 40^{\circ} & 40 \% \\ 54 & 8 & 10^{\circ} & 10 \% \\ 55 & 22 & \{3 \overline{12}\} & \{31 \overline{2}\} \\ 60 & 20 & 3 \mathrm{a} . & 2 \mathrm{a} . \\ 60 & 22 & 2 \mathrm{a} . & 3 \mathrm{a} . \\ 63 & 1 & \text { VALOR DE } & \text { VALOR DE I } \\ 65 & 24 & 3 & 4\end{array}$

$\mathrm{Na}$ pagina 49 falta a notação:

(15) V. Goldschmidt - Atlas der Kristallformem, Heidelberg.

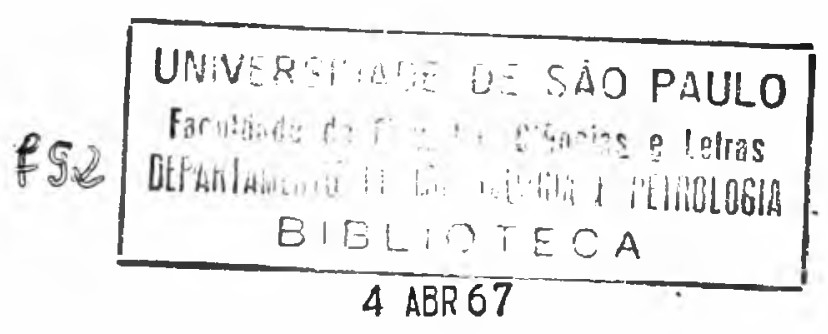

